ARTICLE

DOI: $10.1038 / \mathrm{s} 41467-018-05462-4$

\title{
Facile transformation of imine covalent organic frameworks into ultrastable crystalline porous aromatic frameworks
}

Xinle $\mathrm{Li}^{1}$, Changlin Zhang (10 ${ }^{1}$, Songliang Cai ${ }^{2}$, Xiaohe Lei ${ }^{1,3}$, Virginia Altoe ${ }^{1}$, Fang Hong ${ }^{4}$, Jeffrey J. Urban ${ }^{1}$, Jim Ciston ${ }^{1}$, Emory M. Chan ${ }^{1} \&$ Yi Liu (D) ${ }^{1}$

The growing interest in two-dimensional imine-based covalent organic frameworks (COFs) is inspired by their crystalline porous structures and the potential for extensive $\pi$-electron delocalization. The intrinsic reversibility and strong polarization of imine linkages, however, leads to insufficient chemical stability and optoelectronic properties. Developing COFs with improved robustness and $\pi$-delocalization is highly desirable but remains an unsettled challenge. Here we report a facile strategy that transforms imine-linked COFs into ultrastable porous aromatic frameworks by kinetically fixing the reversible imine linkage via an aza-DielsAlder cycloaddition reaction. The as-formed, quinoline-linked COFs not only retain crystallinity and porosity, but also display dramatically enhanced chemical stability over their iminebased COF precursors, rendering them among the most robust COFs up-to-date that can withstand strong acidic, basic and redox environment. Owing to the chemical diversity of the cycloaddition reaction and structural tunability of COFs, the pores of COFs can be readily engineered to realize pre-designed surface functionality.

\footnotetext{
${ }^{1}$ The Molecular Foundry, Lawrence Berkeley National Laboratory, Berkeley, CA 94720, USA. ${ }^{2}$ School of Chemistry and Environment, South China Normal University, 510006 Guangzhou, China. ${ }^{3}$ Department of Chemistry, Zhejiang University, 310027 Hangzhou, China. ${ }^{4}$ The Advanced Light Source, Lawrence Berkeley National Laboratory, Berkeley, CA 94720, USA. Correspondence and requests for materials should be addressed to Y.L. (email: yliu@lbl.gov)
} 
C ovalent organic frameworks (COFs) are porous, crystalline networks constructed by linking molecular organic building units with covalent bonds ${ }^{1}$. Owing to their high surface area, chemical diversity, and tunable functionality, COFs have been adopted as an auspicious platform for a plethora of applications pertaining to gas adsorption ${ }^{2}$, separation ${ }^{3}$, chemical sensing $^{4}$, catalysis ${ }^{5}$, optoelectronics ${ }^{6}$, and energy storage $^{7}$. In order to establish a covalently linked periodic framework in COFs, current methods rely on the reversible covalent bond formation to connect multivalent monomers through thermodynamic equilibria ${ }^{8,9}$. This inherent reversibility of the linkages within COFs, however, severely limit fundamental materials properties such as environmental stability towards solvents and chemicals, thus constraining their practical applications. For example, COFs with boroxine linkages are susceptible to water or protic solvents ${ }^{10-12}$. COFs based on a more robust linkage, such as the $\mathrm{C}=\mathrm{N}$ imine bond, represent the prevalent class of COFs that show improved hydrothermal stability ${ }^{13}$. The chemical stability of most imine COFs is however still far from satisfactory since they undergo hydrolysis under strongly acidic conditions or exchange with amines due to the reversible nature of imine ${ }^{14}$. As such, a facile methodology that enables the fabrication of chemically robust COFs is much desired ${ }^{15-19}$.

One specific perspective that motivates the field of COFs is the potential of constructing a periodic conjugated 2D framework with covalently linked aromatic ring systems, from which exceptional electronic and magnetic properties are predicted ${ }^{20}$. The imine linkage has been deemed as a better choice than other dynamic bonds as it serves as an $s p^{2}$-hybridized unit readily inserted into a $\pi$ extended framework to open up conjugation pathways. The resulting framework, however, is inferior in facilitating $\pi$ electron delocalization between the linked units on account of the strongly polarized nature of the $\mathrm{C}=\mathrm{N}$ bonds ${ }^{21}$. Despite a few examples of crystalline $\pi$-extended $2 \mathrm{D}$ polymer layers obtained from the surface- or interface-assisted synthesis ${ }^{22,23}$, and a most recent $s p^{2}$ carbon-conjugated $\mathrm{COF}^{24}$, a general strategy to reinforce strong covalent bonding and extended $\pi$-electron delocalization in the same framework remains largely unexplored.

Herein, we explore a strategy that can transform the dynamic imine linkages in COFs into more robust and conductive bonds while preserving the topology, crystallinity, and porosity of COFs. Starting from an imine COF can bypass the crystallization problem encountered in irreversible crosslinking, while the linkage transformation can significantly boost the chemical stability and electron delocalization in the resultant COFs. A number of COFs have been modified postsynthetically ${ }^{25-28}$ to give functionally diverse and topologically identical porous materials. The modification reaction happens almost exclusively on the linkers that bear chemically addressable pendant groups, with only one exception in which the reaction targets the imine linkages to transform them into more stable amide linkages ${ }^{29}$. Herein, we report a more versatile approach to kinetically fix the reversible linkage in imine COFs to afford crystalline and chemically robust porous $2 \mathrm{D}$ aromatic framework with extended $\pi$-electron delocalization (Fig. 1a). Thanks to the efficient Povarov (aza-Diels-Alder, aza-DA) reaction ${ }^{30}$ between aryl imines and arylalkynes, the imine linkages in 2D COFs can be converted to yield the corresponding quinoline-linked COFs (denoted as MF-1a-e). Owing to the structural tunability of starting imine-linked COFs and substrate diversity of the aza-DA reaction, a large variety of functional moieties can be introduced to selectively alter the pore surface and wettability of the resultant COFs.

\section{Results and discussion}

Synthesis of the MFs via aza-DA reaction. The viability of the Povarov reaction was first demonstrated on a prototype imine- linked COF, a highly crystalline and porous COF denoted as COF- $1^{31}$. COF-1 was readily obtained following a reported protocol and was subjected to the reaction with phenylacetylene (1a) at $110^{\circ} \mathrm{C}$ in the presence of $\mathrm{BF}_{3} \cdot \mathrm{Et}_{2} \mathrm{O}$ (1.5 equiv. per imine functionality) and chloranil in toluene for $72 \mathrm{~h}$. The solid separated from the reaction mixture was washed with excess anhydrous THF and saturated $\mathrm{NaHCO}_{3}$, and then dried under vacuum to afford MF-1a as dark yellow solid.

Characterizations of the MFs. The successful addition of phenylacetylene onto COF-1 was verified by several analytical methods. Comparison of the Fourier-transform infrared (FT-IR) spectra of MF-1a and COF-1 (Fig. 2a) revealed a new cluster of peaks at $\sim 1600 \mathrm{~cm}^{-1}$ that correspond to the stretch of aromatic quinoline core ${ }^{30}$ and blue shift of the $\mathrm{C}-\mathrm{C}=\mathrm{N}-\mathrm{C}$ stretch (from 1202 to $1222 \mathrm{~cm}^{-1}$ ) after the reaction. As a control, the 1D iminepolymer (Supplementary Figure 1) was subjected to Povarov reaction to afford modified quinoline-linked polymer, which revealed a similar cluster of peaks at $\sim 1600 \mathrm{~cm}^{-1}$ arising from the aromatic quinoline core and blue shift of the $\mathrm{C}-\mathrm{C}=\mathrm{N}-\mathrm{C}$ stretch (from 1207 to $1214 \mathrm{~cm}^{-1}$ ). Moreover, the $\mathrm{C}-\mathrm{C}=\mathrm{N}-\mathrm{C}$ stretching is close to the model compound, diphenyl quinoline $\left(1238 \mathrm{~cm}^{-1}\right)$. Another control experiment run under the identical reaction conditions except without phenylacetylene 1a confirmed that the Lewis acid catalyst $\left(\mathrm{BF}_{3} \cdot \mathrm{Et}_{2} \mathrm{O}\right)$ and chloranil alone induced no chemical modification of COF-1, as revealed by the lack of evident changes in the FT-IR spectra of COF-1 (Supplementary Figure 2). Given that the quinoline vibration at $\sim 1622 \mathrm{~cm}^{-1}$ in the spectrum of MF-1a overlaps with $\mathrm{C}=\mathrm{N}$ imine stretch of COF1, 1a was replaced with a para-ester substituted phenylacetylene derivative (1b) to afford MF-1b bearing a characteristic chemical probe. FT-IR spectra of MF-1b revealed a peak at $\sim 1725 \mathrm{~cm}^{-1}$, arising from the carbonyl moiety (Fig. 2a), which is also different from that in the spectra of free $\mathbf{1 b}\left(\sim 1702 \mathrm{~cm}^{-1}\right)$ (Supplementary Figure 3). Solid-state ${ }^{13} \mathrm{C}$ cross polarization magic angle spinning (CP-MAS) NMR spectroscopy revealed an apparent up-field shift of the aromatic and-OMe resonances in the spectrum of MF-1a compared to that of COF-1. Such up-field shift is again observed in the spectra of MF-1b, together with two well-resolved but characteristic peaks at 165 and $50 \mathrm{ppm}$ that could be assigned to the methyl ester groups (Fig. 2b). These spectroscopic changes corroborate well with successful conversion of the imine linkages of the COF framework following the Povarov cycloaddition.

The powder X-ray diffraction (PXRD) pattern of COF-1 exhibited six prominent diffraction peaks, with the most intensive one at $2.73^{\circ}$ and the five other peaks at $4.79^{\circ}, 5.54^{\circ}, 7.35^{\circ}, 9.65^{\circ}$, and $25.2^{\circ}$, assigned to the (100), (110), (200), (210), (220), and (001) facets, respectively (Fig. 2c, black curve). After modification, the PXRD pattern of MF-1a exhibited four prominent diffraction peaks, with the most intensive one at $2.75^{\circ}$ and the three other peaks at $4.82^{\circ}, 5.58^{\circ}$, and $25.1^{\circ}$, corresponding to the (100), (110), (200), and (001) facets, respectively (Fig. 2c, red curve). The (100) peaks of MF-1a and MF-1b have a small full-width at halfmaximum (FWHM) values of $0.25^{\circ}$ and $0.29^{\circ}$, respectively, suggesting their high crystallinity. Such peaks show insignificant shift compared to that of COF-1 (FWHM =0.24 $4^{\circ}$ (Fig. 2c), indicating preservation of the crystalline framework during the linkage transformation. Using an optimized monolayer structure, $\mathrm{AA}$ and staggered $\mathrm{AB}$ stacking modes were generated and optimized. The simulated PXRD pattern of the modeled 2D frameworks in AA stacking mode is in good agreement with the experimental peak positions. The PXRD pattern of MF-1a and MF-1b demonstrated similar high crystallinity and insignificant shift of (100) and (001) peaks as compared with that of COF-1 (Fig. 2c), indicating the preservation of the crystalline framework 
a

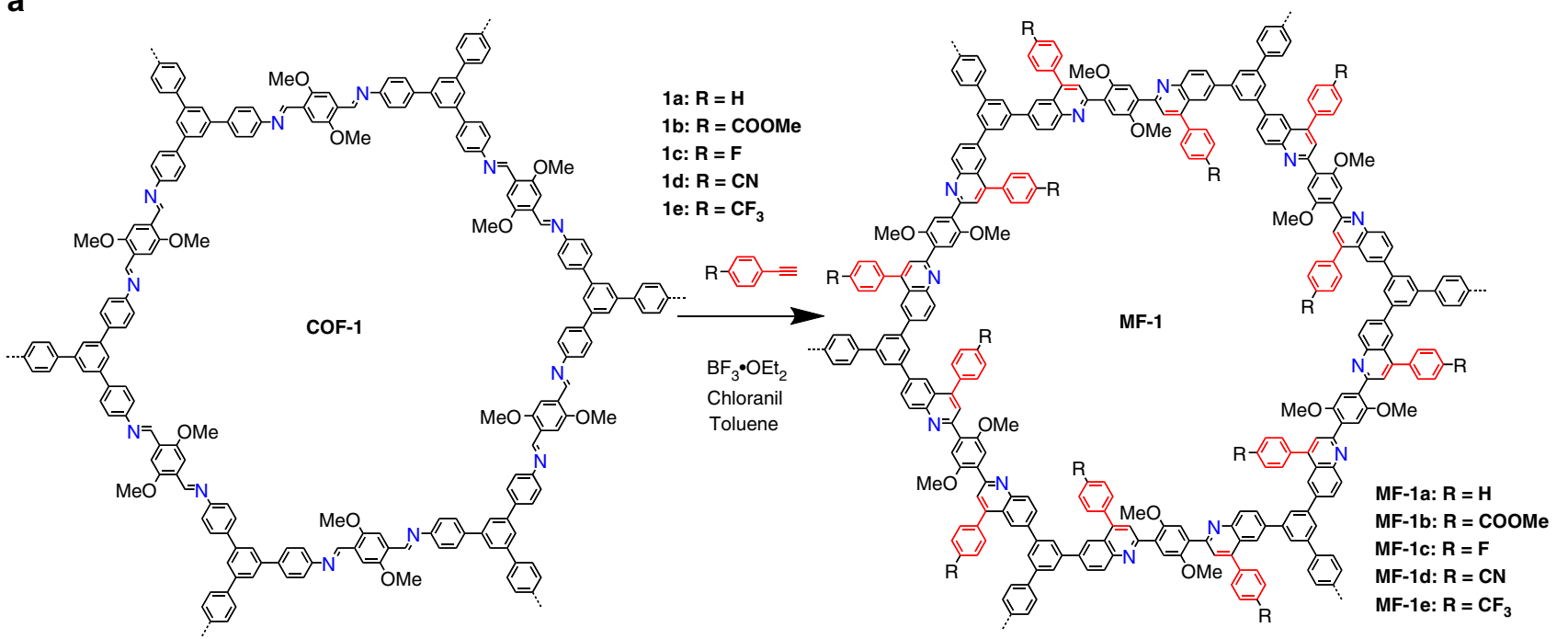

b
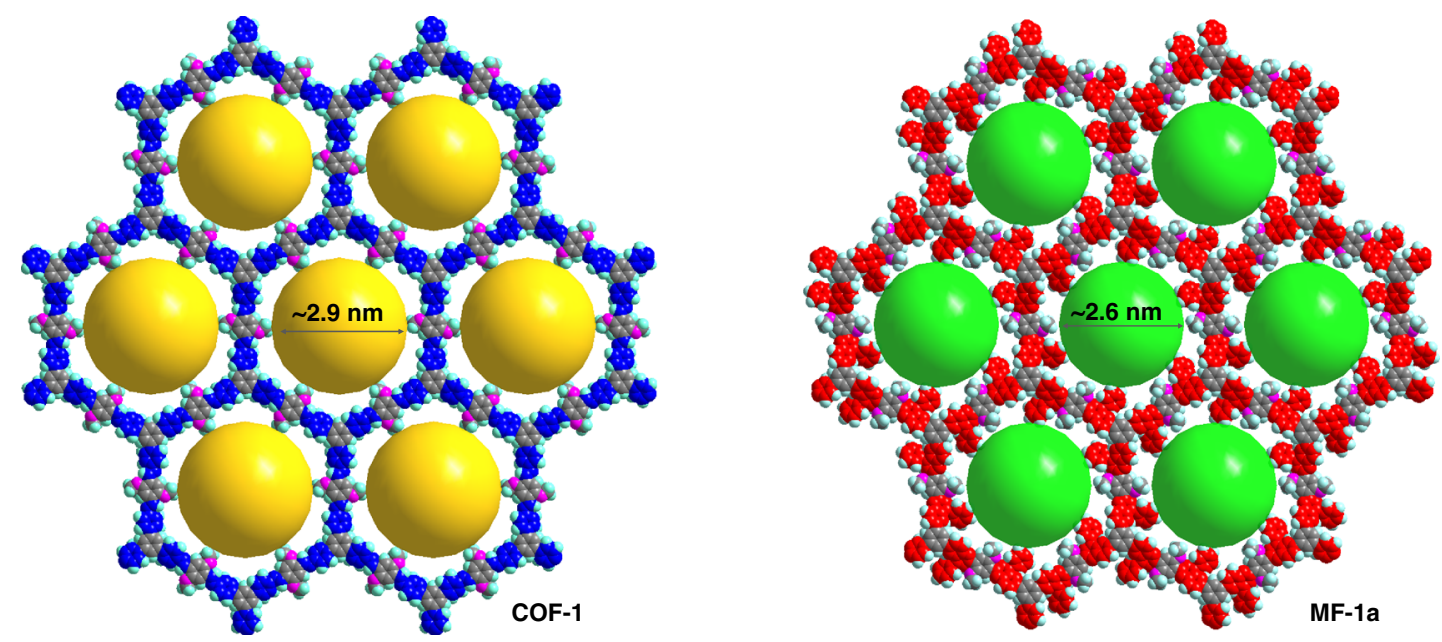

Fig. 1 Post-synthetic modification of COFs via aza-DA reaction. a The reaction scheme showing the transformation of one lattice unit of COF-1 into MF-1ae. $\mathbf{b}$ The simulated modeled structure showing the extended frameworks of COF-1 and MF-1a. The yellow and cyan sphere indicate pore diameter of $\sim 2.9$ and $\sim 2.6 \mathrm{~nm}$. Note that the illustrated structure of MF-1 contains fully converted quinolines and does not represent the actual degree of transformation

during the linkage transformation. Such patterns also matched with the simulated PXRD pattern of the modeled 2D frameworks in AA stacking mode (Fig. $1 \mathrm{~b}$ and Supplementary Figure 4c). Pawley refinement showed the negligible difference between the simulated XRD and experimental patterns (Supplementary Figure $4 \mathrm{~b}$ ), while the staggered $\mathrm{AB}$ stacking mode (Supplementary Figure 4d) did not reproduce the experimental profile (Supplementary Tables 1-5). UV-Vis diffuse reflectance spectra of MF-1a (Supplementary Figure 5a, red curve) showed a red shift as compared with COF-1 (Supplementary Figure 5a, black curve), which is indicative of enhanced $\pi$ delocalization. Upon modification, MF-1a showed a reduced optical bandgap of 2.30 $\mathrm{eV}$ compared to that of COF-1 $(2.52 \mathrm{eV})$. As shown by thermogravimetric analysis (Supplementary Figure 6), MF-1a has a slightly higher decomposition onset temperature compared to COF-1. In addition, it displays less weight loss than COF-1 after incubation at $400^{\circ} \mathrm{C}$, indicating better thermal stability after the chemical transformation.

$\mathrm{N}_{2}$ sorption analyses performed at $77 \mathrm{~K}$ revealed a Brunauer -Emmett-Teller (BET) surface area of 1760, 955, and $590 \mathrm{~m}^{2} \mathrm{~g}^{-1}$ for COF-1, MF-1a, and MF-1b, respectively (Fig. 2d). The decrease of surface area corroborates with the increased framework mass and reduction of the pore volume. The type IV isotherms of MF-1a, MF-1b, and COF-1 are not only indicative of the mesoporous characters, but also suggest that the chemical modification does not lead to significant changes in the framework structure. Pore size distribution analysis indicated a pore size reduction from 3.0 to $2.1 \mathrm{~nm}$ upon chemical transformation (Supplementary Figure 7). Additional SEM studies showed that the morphology of MF-1a is similar to that of the pristine COF-1 (Supplementary Figure 8). X-ray photoelectron spectroscopy (XPS) analyses were performed to provide more insight into the conversion from imine to quinoline. As shown in Supplementary Figure 9, the N1s peak at $\sim 398.0 \mathrm{eV}$ in COF-1 can be attributed to the imine $\mathrm{N}$ atoms ${ }^{32}$. Upon modification, a fraction of the N1s core level is shifted to higher binding energies $(\sim 399.5 \mathrm{eV})$, corresponding to the $\mathrm{C}=\mathrm{N}$ in quinoline moieties ${ }^{33}$. Based on the integration of the peak areas, the degrees of functionalization in MF-1a and MF-1b are assessed to be $27 \%$ and $25 \%$, respectively. Further XPS depth profiling analysis indicates that the quinoline/ imine nitrogen ratio remains constant after repeated exposure to high energy argon ion beam irradiations, confirming a uniform material composition from the surface to the buried body of the bulk (Supplementary Figure 10). In the case of MF-1a, increasing the reaction time to 1 week resulted in a slight increase of the conversion to $29 \%$, while increasing the concentration of all the 

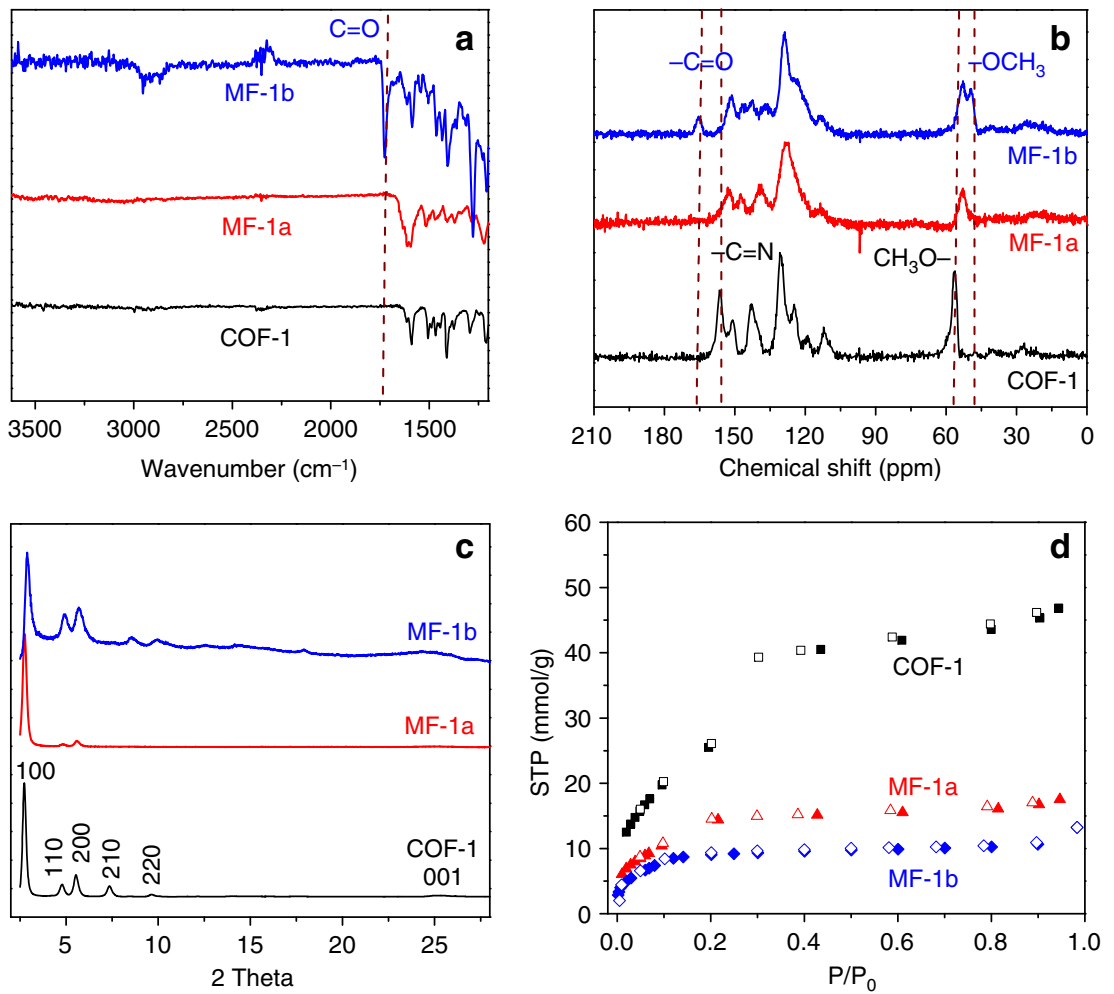

Fig. 2 Characterization of original COF-1 (black) and post-synthetically modified MF-1a (red) and MF-1b(blue). a FT-IR spectra. b Solid-state ${ }^{13}$ C CP-MAS NMR spectra. c PXRD patterns showing retention of crystallinity after the Povarov reaction. $\mathbf{d} \mathrm{N}_{2}$ sorption isotherm curves

reactants except COF-1 by 3 -fold augmented the conversion to $35 \%$ (Supplementary Figure 11), suggesting that higher conversion may be achieved by further optimization of the reaction conditions.

The periodic framework structural features of COF-1 and MF1a were visualized by high-resolution transmission electron microscopy (HRTEM), using the low-dose TEM technique equipped with Gatan K2 Summit direct-detection electroncounting camera ${ }^{34}$ and geometric/chromatic image aberration correction. The total electron dose was 2.1 e $\AA^{-2}$ and the pixel size $0.72 \AA$. As shown in Fig. 3a, the honeycomb-like porous structure of COF-1 was clearly observed along the [111] direction, with the pore opening determined as $3.0 \pm 0.2 \mathrm{~nm}$. Based on the Fourier-filtered image of a selected area (red square in Fig. 3a and Supplementary Figure 12), we can clearly observe a hexagonal projected symmetry constructed by six white diffraction spots. Upon modification, MF-1a shows a decreased pore opening of $2.3 \pm 0.2 \mathrm{~nm}$ (Fig. 3c) with the preservation of similar honeycomb-like porous structure (Fig. 3b, d). The direct observation of the pore opening decrease in the framework structures before and after modification, which is consistent with the modeled structures as shown in Fig. $1 \mathrm{~b}$ and pore size distributions (Supplementary Figure 7), confirmed the effectiveness of the framework-to-framework transformation. Note that COF samples are highly electron-beam sensitive, making the acquisition of high-resolution extremely challenging ${ }^{35-37}$, this work represents a rare example in which the individual building units of COFs are directly and clearly observed by HRTEM.

Chemical stability. To assess the chemical stability of the modified framework structure, MF-1a was subjected to a variety of extremely harsh chemical conditions. The post-synthetically

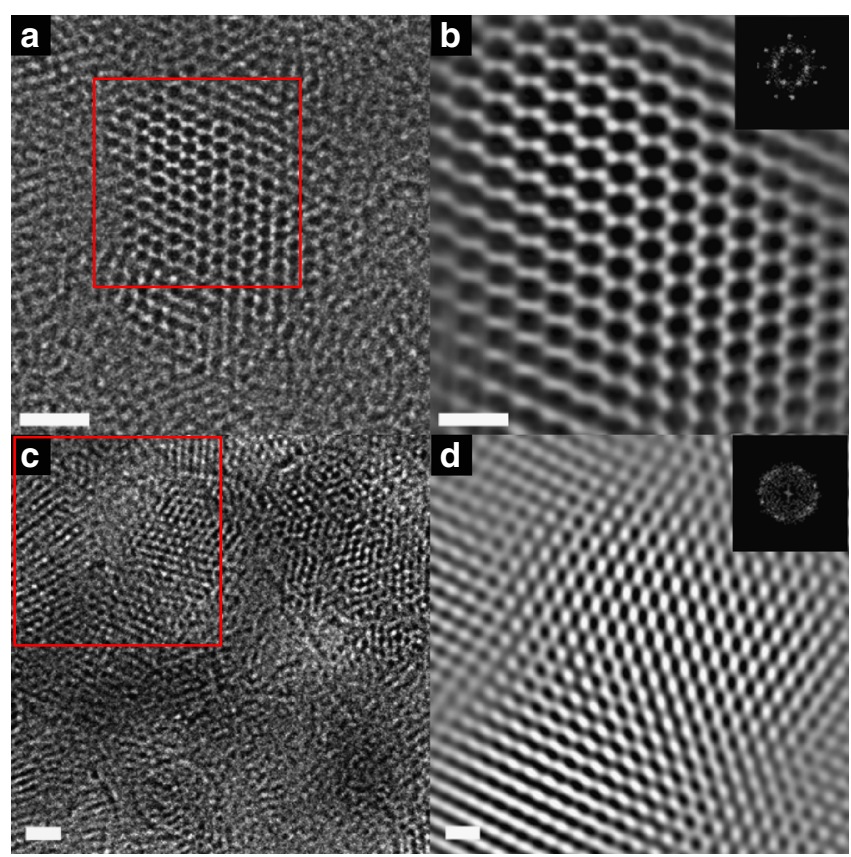

Fig. 3 HRTEM characterization of COF-1 and MF-1a. a Low-dose, highresolution TEM image of COF-1 (scale bar, $10 \mathrm{~nm}$ ). b The Fourier-filtered image of selected red square areas (scale bar, $5 \mathrm{~nm}$ ), Inset: Fast Fourier Transform (FFT) from the red square on the COF-1. c Low-dose, highresolution TEM image of MF-1a (scale bar, $10 \mathrm{~nm}$ ). d The Fourier-filtered image of the selected red square area (scale bar, $5 \mathrm{~nm}$ ), Inset: FFT from the red square on the MF-1a 

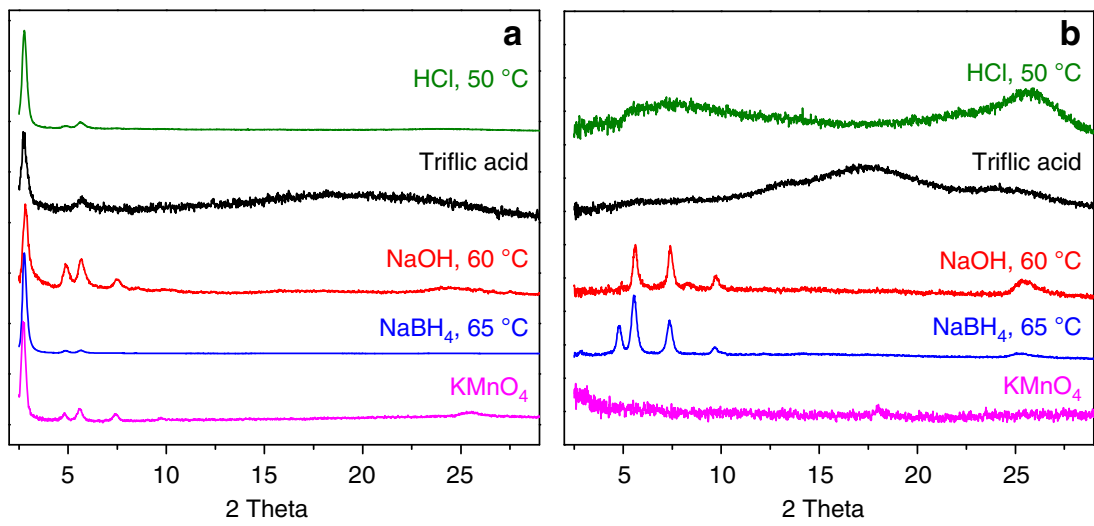

Fig. 4 Chemical stability of MF-1a and COF-1. PXRD patterns of MF-1a (a) and COF-1 (b) after treatment with $12 \mathrm{M} \mathrm{HCl}$ at $50^{\circ} \mathrm{C}$ for $8 \mathrm{~h} \mathrm{(green),} 98 \%$ triflic acid at ambient temperature for 3 days (black), $14 \mathrm{M} \mathrm{NaOH}$ in $\mathrm{H}_{2} \mathrm{O} / \mathrm{MeOH}$ solution at $60^{\circ} \mathrm{C}$ for 1 day (red), 5 equiv. of $\mathrm{NaBH}_{4}$ in $\mathrm{MeOH}$ at $65^{\circ} \mathrm{C}$ for 1 day (blue), and 5 equiv. of $\mathrm{KMnO}_{4}$ in $\mathrm{H}_{2} \mathrm{O} / \mathrm{CH}_{3} \mathrm{CN}$ solution at ambient temperature for 1 day (purple)

modified COF exhibited strikingly high chemical stability in strong mineral acid $\left(12 \mathrm{M} \mathrm{HCl}\right.$ at $50^{\circ} \mathrm{C}$ for $8 \mathrm{~h}$ ), superacid $(98 \% \mathrm{TfOH}$, 3 days), strong base ( $14 \mathrm{M} \mathrm{NaOH}$ in $\mathrm{H}_{2} \mathrm{O} / \mathrm{MeOH}$ at $60^{\circ} \mathrm{C}, 1$ day), strong oxidant $\left(\mathrm{KMnO}_{4}\right.$ in $\mathrm{H}_{2} \mathrm{O} / \mathrm{CH}_{3} \mathrm{CN}$ solution, 1 day) and reducing agents $\left(\mathrm{NaBH}_{4}\right.$ in $\mathrm{MeOH}$ at $65^{\circ} \mathrm{C}, 1$ day), as revealed by the retention of major diffraction peaks in the PXRD patterns of the treated samples (Fig. 4a). Remarkably, MF-1a retained its crystallinity after being exposed to $12 \mathrm{M} \mathrm{HCl}$ at room temperature for 2 months (Supplementary Figure 13), or to boiling acids (1 M and $12 \mathrm{M} \mathrm{HCl})$ and bases $(1 \mathrm{M}$ and $14 \mathrm{M} \mathrm{NaOH})$ for 1 day (Supplementary Figure 14). IR spectra of the acid-treated MF-1a indicated the appearance of aldehyde vibration band at $\sim 1670 \mathrm{~cm}^{-1}$, suggesting partial hydrolysis of the remaining imine bonds under such forcing conditions (Supplementary Figure 15). As the porous framework is well preserved due to the introduced quinoline linkage, the hydrolysis conceivably gives a patchy framework that is decorated with dangling aldehyde and amine functional groups, which present further opportunities for introducing extra functionality. Such a high framework stability compares favorably to other known stable framework materials (Supplementary Table 6). In sharp contrast, the corresponding COF- 1 , one of the most chemically robust imine COFs reported up-to-date, is rendered amorphous or loses framework periodicity (Fig. $4 \mathrm{~b}$ and Supplementary Figures 13, 14). The residual weight percentages of MF-1a under the conditions of strong acid (12 M $\mathrm{HCl}$ at $\left.50^{\circ} \mathrm{C}\right)$ and strong base $\left(14 \mathrm{M} \mathrm{NaOH}\right.$ at $\left.60^{\circ} \mathrm{C}\right)$ are 88 and $83 \mathrm{wt} \%$ while the pristine COF-1 exhibited more significant weight loss after acid/base treatment (Supplementary Figure 16). Moreover, MF-1a still remained porous with only 5-25\% decrease in surface area. whereas the pristine COF-1 displayed much more significant loss $(80-100 \%)$ in surface area (Supplementary Figure 17). The vast differential in the stability of the MF-1 library relative to COF-1 clearly underscores the significance of transforming dynamic imines to much more robust quinoline units. The greatly enhanced stability towards chemical oxidation and reduction is particularly remarkable and is relevant for practical applications involving redox processes.

Generality of the Povarov addition approach. The cycloaddition-based approach allows the integration of various functionalities onto the pores of COFs from substituted arylalkynes, thus rendering it much more versatile than the oxidationbased post-synthetic modification of imine-COFs ${ }^{29}$, and click reaction-based post-synthetic modification of $\mathrm{COFs}$, which requires specifically designed azide/ethynyl-appended building blocks and metal catalyst (e.g., CuI $)^{25,26}$. The generality was demonstrated by reacting COF-1 with para-substituted arylalkynes 1c-e under similar reaction conditions to give MF-1c-e bearing fluorine, nitrile and trifluoromethyl groups on the pore surfaces. The PXRD analysis confirmed the retention of crystallinity after the Povarov reaction (Fig. 5a) while solid-state CPMAS ${ }^{13} \mathrm{C}$ NMR (Supplementary Figure 18) and FT-IR spectroscopy revealed changes that correspond to the conversion of imine bonds to quinoline $\mathrm{C}=\mathrm{N}$ bonds (Fig. 5b). Notably, the characteristic $-\mathrm{CN}$ vibrational peak $\left(\sim 2231 \mathrm{~cm}^{-1}\right)$ and $-\mathrm{CF}_{3}$ stretch $\left(\sim 1325 \mathrm{~cm}^{-1}\right)$ in the FT-IR spectra of MF-1b and MF-1c, which are different from these in the spectra of free 1d $\left(2226 \mathrm{~cm}^{-1}\right)$ and 1e $\left(1320 \mathrm{~cm}^{-1}\right)$ (Supplementary Figure 19), together with the absence of alkyne peaks $\left(\sim 3200-3300 \mathrm{~cm}^{-1}\right.$ for $-\mathrm{C} \equiv \mathrm{C}-\mathrm{H}$ stretch and $\sim 2100 \mathrm{~cm}^{-1}$ for $-\mathrm{C} \equiv \mathrm{C}$ - stretch), unambiguously confirmed the chemical attachment of $-\mathrm{PhCN}$ and $-\mathrm{PhCF}_{3}$ groups to the framework (highlighted in cyan in Fig. $5 \mathrm{~b}$ ) that was concomitant with successful Povarov reaction. XPS analysis shows the degree of functionalization for MF-1 c-e is $27 \%, 29 \%$, and $26 \%$, respectively (Supplementary Figure 9), very similar to that of MF-1 a-b.

This post-synthetic modification protocol can be applied to other imine-COFs as well, as demonstrated in the reaction with COF-2 (Supplementary Figure 20), which was readily synthesized following a routinesolvothermal procedure ${ }^{38}$. The disappearance of the characteristic imine stretch at $1625 \mathrm{~cm}^{-1}$ is clearly discernible in the FT-IR spectra of MF-2a (Supplementary Figure 21). The retention of morphology, crystallinity, and porosity in MF-2a was confirmed by SEM images (Supplementary Figure 22), PXRD analysis (Supplementary Figure 23) and $\mathrm{N}_{2}$ sorption analysis (Supplementary Figure 24), respectively. The enhanced chemical stability was demonstrated by treating MF-2a with a strong acid $(12 \mathrm{M} \mathrm{HCl}, 1$ day), the PXRD of which remained unchanged while COF-2 decomposed completely under the same conditions (Supplementary Figure 25). Finally, a variety of organic functionalities such as $-\mathrm{F},-\mathrm{CN}$, and $-\mathrm{CF}_{3}$ groups have been successfully incorporated into the porous crystalline framework via the Povarov reaction of COF-2, as verified by PXRD and FT-IR analyses (Supplementary Figure 26). XPS analysis indicates the degree of functionalization in MF-2 a-d is $21 \%, 29 \%, 24 \%$, and $29 \%$, respectively (Supplementary Figure 27), which is comparable to the MF-1 series. All these results correlate well with a successful chemical transformation of imine-COFs into more stable crystalline porous frameworks.

Customizable surface wettability. The customizable surface functionality of stable COFs provides a convenient way to 
a

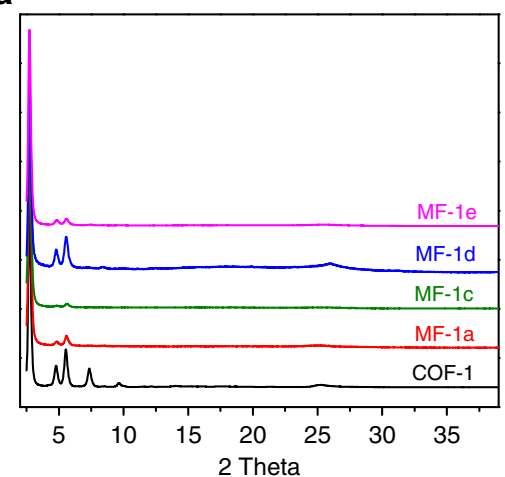

b

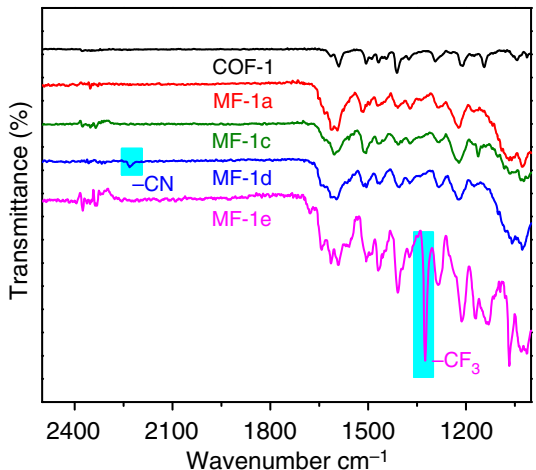

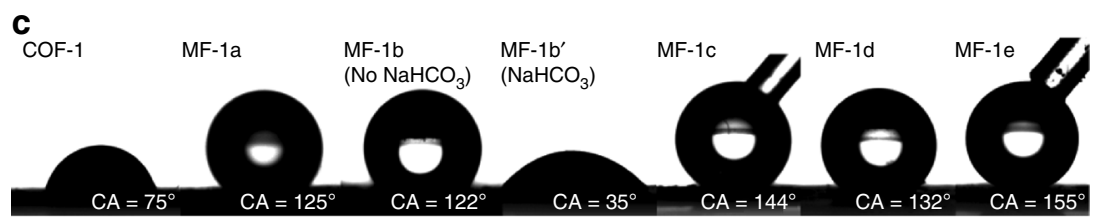

Fig. 5 Structural characterization and surface properties of MF-1 series. a Powder XRD patterns of COF-1 (black), MF-1a (red), MF-1c (green), MF-1d (blue), and MF-1-e (purple). b FT-IR spectra of COF-1 and MF-1a, c-e. The peaks highlighted in cyan are characteristic vibrations from these functional groups. c Water contact angles (CA) of water droplet on the pressed pellet of COF-1 and MF-1a-e. MF-1b' is the NaHCO $\mathrm{N}_{3}$-treated MF-1b that undergoes partial ester hydrolysis

fabricate porous materials with controlled wettability, which has garnered continuous attention from both academia and industry ${ }^{39-41}$. The surface wettability of COFs before and after the Povarov reaction differs drastically and shows a strong dependence on both the COF structure and the substituent groups on the phenylacetylene. Figure $5 c$ shows water contact angles on the surface of COF-1 and MF-1a-e. The contact angle on MF-1a is $\sim 125^{\circ}$ while COF- 1 exhibits a contact angle of only $\sim 75^{\circ}$, indicating that the covalent modification of COFs significantly enhanced the hydrophobicity of the material. This increase in hydrophobicity is presumably due to the introduction of more hydrophobic aromatic rings onto the pore surfaces and decreased polarization after incorporating the imine bonds into the aromatic quinoline ring systems. Notably, incremental changes of contact angles are observed when altering the functional groups from $-\mathrm{H}\left(\mathrm{MF}-1 \mathrm{a}, 125^{\circ}\right)$ to $-\mathrm{CN}\left(\mathrm{MF}-1 \mathrm{~d}, 132^{\circ}\right),-\mathrm{F}\left(\mathrm{MF}-1 \mathrm{c}, 144^{\circ}\right)$, and $-\mathrm{CF}_{3}\left(\mathrm{MF}-1 \mathrm{e}, 155^{\circ}\right)$, highlighting an effective way to systematically fine-tune COF surface wettability into the superhydrophobic region. The methyl ester-bearing $\mathrm{MF}-1 \mathrm{~b}$ has a similar contact angle of $122^{\circ}$. Upon treatment with $\mathrm{NaHCO}_{3}$, the contact angle $\left(\mathrm{MF}-1 \mathrm{~b}^{\prime}\right)$ decreases significantly to $35^{\circ}$, indicating the feasibility of imparting hydrophilicity via hydrolysis of ester moieties. Similar fine modulation of hydrophobicity is also observed in the series of MF-2s. MF-2a has a contact angle of $152^{\circ}$ which is significantly higher than the $125^{\circ}$ contact angle of the pristine COF-2 and the introduction of fluorinated functional groups $\left(-\mathrm{F}\right.$, and $\left.-\mathrm{CF}_{3}\right)$ led to gradually increased hydrophobicity, evidenced by the contact angle change from $-\mathrm{H}\left(\mathrm{MF}-2 \mathrm{a}, 152^{\circ}\right)$ to $-\mathrm{F}\left(\mathrm{MF}-2 \mathrm{c}, 157^{\circ}\right)$ and $-\mathrm{CF}_{3}\left(\mathrm{MF}-2 \mathrm{e}, 162^{\circ}\right)$ (Supplementary Figure 28).

We have developed a facile approach to deriving crystalline porous aromatic frameworks from readily available imine-COFs by transforming the dynamic imine linkages into more stable quinoline aromatic ring systems via aza-Diels-Alder cycloaddition. The kinetic fixation of the imine linkages, even with only around $20-30 \%$ conversion, resulted in dramatically enhanced framework stability towards strong acid, base, and redox reagents. This framework-to-framework transformation offers a simple solution to the intrinsic instability associated with imine-COFs while retaining the framework's crystallinity and permanent porosity, while simultaneously enabling the tuning of pore surface functionalities and $\pi$ electron delocalization. This succinct protocol paves the way to the synthesis of crystalline, porous aromatic frameworks that are difficult to obtain de novo, and it will facilitate practical applications of organic framework materials that require enhanced chemical stability, semiconducting properties, and pore surface functionality.

\section{Methods}

Reagents. Acetone, acetonitrile, chloroform, dichloromethane, methanol, tetrahydrofuran (THF), toluene, and 1,4-dioxane were purchased from Fisher chemicals. 1-ethynyl-4-fluorobenzene and 1,3,5-tri-(4-aminophenyl)benzene were purchased from TCI. 1-ethynyl-4-(trifluoromethyl)benzene as purchased from 1Click Chemistry Stock Products. Methyl 4-ethynyl benzoate was purchased from AK scientific. 2,5-Dimethoxyterephthalaldehyde were purchased from Carbosynth chemicals. 2,4-diphenyl-quinoline were purchased from EnamineStore. Phenylacetylene, 4-ethynyl benzonitrile, boron trifluoride diethyl etherate, triethylamine were purchased from Alfa Aesar chemicals. Chloranil, triflic acid, 1,3,5-triformylbenzene, benzidine, mesitylene, $o$-dichlorobenzene, $n$-butanol were purchased from Sigma Aldrich chemicals.

\section{Instrumentation and characterization. Fourier transform infrared (FT-IR)} spectra were recorded on a Perkin Elmer Spectrum One FT-IR system. Powder Xray diffraction (PXRD) patterns were recorded on a Bruker Discovery D8 X-ray diffractometer and Rigaku MiniFLex 6G Benchtop XRD with $\mathrm{Cu}$ Kal radiation $(\lambda=1.5406 \AA)$. Nitrogen sorption isotherms were obtained at $77 \mathrm{~K}$ with a Micromeritics Instrument Corporation model 3Flex surface characterization analyzer. The Brunauer-Emmett-Teller (BET) method was utilized to calculate the specific surface areas. By using the non-local density functional theory (NLDFT) model, the pore size distribution was derived from the sorption curve. TGA measurements were performed on a TA Instruments Q5000IR TGA under Argon, by heating to $600^{\circ} \mathrm{C}$ at a rate of $10^{\circ} \mathrm{C} \mathrm{min}{ }^{-1}$. Solid-state ${ }^{13} \mathrm{C}$ CP-MAS NMR spectra were recorded on a BrukerAvance500 I (MF-1d sample only) and BrukerAvance500 II. The UV-vis diffuse reflectance measurement was performed on Cary 5000 UV-VisNIR spectrometer. X-ray photoelectron spectroscopy (XPS) measurement was carried out on a Thermo Scientific K-Alpha XPS apparatus equipped with a monochromatic $\mathrm{Al} \mathrm{K}$ (alpha) source and food gun for charge compensation. To remove the acid residues, the COFs samples were treated with triethylamine methanolic solution, followed by washing with copious amount of methanol and degas at $200^{\circ} \mathrm{C}$ under dynamic vacuum for 1 day prior to XPS measurement. Lowdose TEM images of COF-1 were acquired on the TEAM I FEI Titan-class microscope at $300 \mathrm{kV}$, equipped with both geometric aberrations corrected to third order and chromatic aberrations corrected to the first order. Imaging data were collected in the Gatan K2 direct-detection camera operated in electron-counting 
mode (camera counting frame rate of $400 \mathrm{fps}$ (frames per second) at $4 \mathrm{k} \times 4 \mathrm{k}$ resolution) with a final image output rate of $40 \mathrm{fps}$ at $4 \mathrm{k} \times 4 \mathrm{k}$ resolution. The HRTEM image of MF-1a was acquired with an FEI TitanX 60300 microscope at $200 \mathrm{kV}$. The COF sample was sonicated in toluene with a sonication probe for 15 min and drop-casted onto a copper grid (Lacey $\mathrm{C}$ only, 300 mesh $\mathrm{Cu}$ ). SEM images were obtained with a Zeiss Gemini Ultra-55 Analytical Field Emission Scanning Electron Microscope operated at $15 \mathrm{kV}$ using an in-lens detector. Water contact angle measurements were carried out using a Kruss easy drop optical contact angle meter (Model: FM41) under ambient conditions.

Crystal structure modeling. Since the framework structures of MF-1a-e are quite similar, and those of MF-2a-e are close as well, thus only MF-1a and MF-2a are selected as representative examples for structure modeling. The crystal models for MF-1a and MF-2a including the cell parameters and the atomic positions were produced by Materials Studio 5.0 software packagel employing the Crystal Building module. The Pawley PXRD refinement was conducted using the Reflex module in the Materials Studio 5.0, in which a Pseudo-Voigt profile function was employed for whole profile fitting (peak broadening, peak asymmetry, and zero shift error were taken into account). Unit cell and sample parameter were refined simultaneously. The Pawley refinement results including unit cell parameters and final related refinement factors for MF-1a and MF-2a were listed in Supplementary Table 1, while the atomic coordinates of MF-1a and MF-2a crystal structure models are presented in Supplementary Tables $2-5$

Synthesis of COF-1. An $o$-dichlorobenzene $/ n$-butanol $(1 \mathrm{~mL} / 1 \mathrm{~mL})$ mixture of 1,3,5-tri-(4-aminophenyl) benzene $(56 \mathrm{mg}, 0.16 \mathrm{mmol}$ ) and 2,5-dimethoxy terephthalaldehyde $(46 \mathrm{mg}, 0.24 \mathrm{mmol})$ in the presence of acetic acid $(6 \mathrm{M}, 0.2 \mathrm{~mL})$ in a Biotage microwave vial $(5 \mathrm{~mL})$ was degassed through 3 freeze-pump-thaw cycles. The vial was sealed and heated at $120^{\circ} \mathrm{C}$ for 3 days. The precipitate was collected via centrifugation, washed times with anhydrous THF and subjected to Soxhlet extraction using THF as the solvent for 1 day. The powder collected was dried at $120^{\circ} \mathrm{C}$ under vacuum overnight to give yellow colored COF-1 in an isolated yield of $\sim 80 \%$.

Synthesis of COF-2. Benzidine ( $64 \mathrm{mg}, 0.2 \mathrm{mmol}$ ) and 1,3,5-triformylbenzene (32 $\mathrm{mg}, 0.15 \mathrm{mmol}$ ) were dissolved via sonication in a mixture of 1,4-dioxane/ mesitylene $(1 \mathrm{~mL} / 1 \mathrm{~mL})$ in a $5 \mathrm{~mL}$-Biotage microwave vial. Afterwards, aqueous acetic acid $(6 \mathrm{M}, 0.2 \mathrm{~mL})$ was added to the mixture. The vial was degassed by three freeze-pump-thaw cycles. Finally, the vial was sealed and heated in an oven at $120^{\circ} \mathrm{C}$ for 3 days. The precipitates were isolated by centrifugation, washed with anhydrous THF for three times, and dried at $120^{\circ} \mathrm{C}$ under vacuum overnight to give a yellow colored powder in $\sim 90 \%$ yield.

Synthesis of MFs via Povarov reaction. COFs $(4 \mathrm{mg})$, phenylacetylene $(6 \mu \mathrm{L}$, $0.05 \mathrm{mmol}), \mathrm{BF}_{3} \cdot \mathrm{OEt}_{2}(4 \mu \mathrm{L}, 0.03 \mathrm{mmol})$, chloranil $(8 \mathrm{mg}, 0.03 \mathrm{mmol})$, and $2 \mathrm{~mL}$ of toluene were added to a $5 \mathrm{~mL}$-Biotage microwave vial. The vial was sealed and heated under $\mathrm{N}_{2}$ at $110^{\circ} \mathrm{C}$ in an oil bath. After 1-3 days, the mixture was cooled to room temperature and the precipitate was isolated via centrifugation. The reaction mixture was then washed with THF and quenched with saturated aqueous sodium bicarbonate $(2 \mathrm{~mL} \times 3)$. Subsequently, the solids were washed with THF using a Soxhlet extractor for $12 \mathrm{~h}$ and dried under vacuum.

Chemical stability test of MF-1a. The MF-1a sample ( $2 \mathrm{mg})$ was kept for pre designed time under static condition in $0.5 \mathrm{~mL}$ of $98 \%$ triflic acid (2\% catalytic $\left.\mathrm{H}_{2} \mathrm{O}\right)$ at ambient temperature, $0.5 \mathrm{~mL} \mathrm{HCl}(1$ and $12 \mathrm{M})$ at ambient temperature, $50{ }^{\circ} \mathrm{C}$ or $100{ }^{\circ} \mathrm{C}, \mathrm{NaOH}(1$ and $14 \mathrm{M})$ in $\mathrm{H}_{2} \mathrm{O} /$ methanol solution at $60^{\circ} \mathrm{C}$ or $100^{\circ} \mathrm{C}$, $\mathrm{NaBH}_{4}$ (5 equiv. per imine functionality in MF-1a) in methanol at $60^{\circ} \mathrm{C}$ and $\mathrm{KMnO}_{4}$ (5 equiv. per imine functionality in MF-1a) in $\mathrm{H}_{2} \mathrm{O} / \mathrm{CH}_{3} \mathrm{CN}$ solution. The samples were washed with THF for three times, dried under vacuum at ambient temperature and subjected to PXRD measurements.

Data availability. All relevant data supporting the findings of this study are available from the corresponding author on request.

Received: 13 May 2018 Accepted: 6 July 2018

Published online: 31 July 2018

\section{References}

1. Diercks, C. S. \& Yaghi, O. M. The atom, the molecule, and the covalent organic framework. Science 355, eaal1585 (2017).

2. Furukawa, H. \& Yaghi, O. M. Storage of hydrogen, methane, and carbon dioxide in highly porous covalent organic frameworks for clean energy applications. J. Am. Chem. Soc. 131, 8875-8883 (2009).
3. Oh, H. et al. A cryogenically flexible covalent organic framework for efficient hydrogen isotope separation by quantum sieving. Angew. Chem. Int. Ed. 52, 13219-13222 (2013).

4. Ding, S.-Y. et al. Thioether-based fluorescent covalent organic framework for selective detection and facile removal of mercury (II). J. Am. Chem. Soc. 138 3031-3037 (2016).

5. Lu, S. et al. Synthesis of ultrafine and highly dispersed metal nanoparticles confined in a thioether-containing covalent organic framework and their catalytic applications. J. Am. Chem. Soc. 139, 17082-17088 (2017).

6. Wan, S., Guo, J., Kim, J., Ihee, H. \& Jiang, D. A belt-shaped, blue luminescent, and semiconducting covalent organic framework. Angew. Chem. Int. Ed. 120, 8958-8962 (2008).

7. DeBlase, C. R., Silberstein, K. E., Truong, T.-T., Abruña, H. D. \& Dichtel, W. R. $\beta$-Ketoenamine-linked covalent organic frameworks capable of pseudocapacitive energy storage. J. Am. Chem. Soc. 135, 16821-16824 (2013).

8. Jin, Y., Hu, Y. \& Zhang, W. Tessellated multiporous two-dimensional covalent organic frameworks. Nat. Rev. Chem. 1, 0056 (2017).

9. Huang, N. et al. Multiple-component covalent organic frameworks. Nat. Commun. 7, 12325 (2016)

10. Cote, A. P. et al. Porous, crystalline, covalent organic frameworks. Science $\mathbf{3 1 0}$, 1166-1170 (2005)

11. Cote, A. P., El-Kaderi, H. M., Furukawa, H., Hunt, J. R. \& Yaghi, O. M. Reticular synthesis of microporous and mesoporous 2D covalent organic frameworks. J. Am. Chem. Soc. 129, 12914-12915 (2007).

12. Wan, S., Guo, J., Kim, J., Ihee, H. \& Jiang, D. A photoconductive covalen organic framework: self-condensed arene cubes composed of eclipsed 2D polypyrene sheets for photocurrent generation. Angew. Chem. Int. Ed. $\mathbf{4 8}$ 5439-5442 (2009).

13. Segura, J. L., Mancheño, M. J. \& Zamora, F. Covalent organic frameworks based on Schiff-base chemistry: synthesis, properties and potential applications. Chem. Soc. Rev. 45, 5635-5671 (2016).

14. Qian, C. et al. Toward covalent organic frameworks bearing three different kinds of pores: the strategy for construction and COF-to-COF transformation via heterogeneous linker exchange. J. Am. Chem. Soc. 139, 6736-6743 (2017).

15. Kandambeth, S. et al. Construction of crystalline $2 \mathrm{D}$ covalent organic frameworks with remarkable chemical (acid/base) stability via a combined reversible and irreversible route. J. Am. Chem. Soc. 134, 19524-19527 (2012).

16. Kandambeth, S. et al. Enhancement of chemical stability and crystallinity in porphyrin-containing covalent organic frameworks by intramolecular hydrogen bonds. Angew. Chem. Int. Ed. 125, 13290-13294 (2013).

17. Chen, $\mathrm{X}$. et al. Locking covalent organic frameworks with hydrogen bonds: general and remarkable effects on crystalline structure, physical properties, and photochemical activity. J. Am. Chem. Soc. 137, 3241-3247 (2015).

18. Wei, P.-F. et al. Benzoxazole-linked ultrastable covalent organic frameworks for photocatalysis. J. Am. Chem. Soc. 140, 4623-4631 (2018).

19. Arjun, $\mathrm{H}$. et al. Ultrastable imine-based covalent organic frameworks for sulfuric acid recovery: an effect of interlayer hydrogen bonding. Angew. Chem. Int. Ed. 57, 5797-5802 (2018).

20. Xu, Y., Jin, S., Xu, H., Nagai, A. \& Jiang, D. Conjugated microporous polymers: design, synthesis and application. Chem. Soc. Rev. 42, 8012-8031 (2013).

21. Rao, M. R., Fang, Y., De Feyter, S. \& Perepichka, D. F. Conjugated covalent organic frameworks via michael addition-elimination. J. Am. Chem. Soc. 139, 2421-2427 (2017).

22. Liu, W. et al. A two-dimensional conjugated aromatic polymer via C-C coupling reaction. Nat. Chem. 9, 563 (2017).

23. Liu, J. et al. Solution synthesis of semiconducting two-dimensional polymer via trimerization of carbonitrile. J. Am. Chem. Soc. 139, 11666-11669 (2017).

24. Jin, E. et al. Two-dimensional sp2 carbon-conjugated covalent organic frameworks. Science 357, 673-676 (2017).

25. Nagai, A. et al. Pore surface engineering in covalent organic frameworks. Nat Commun. 2, 536 (2011).

26. Huang, N., Krishna, R. \& Jiang, D. Tailor-made pore surface engineering in covalent organic frameworks: systematic functionalization for performance screening. J. Am. Chem. Soc. 137, 7079-7082 (2015).

27. Khayum, M. A. et al. Chemically delaminated free-standing ultrathin covalent organic nanosheets. Angew. Chem. Int. Ed. 55, 15604-15608 (2016).

28. Lohse, M. S. et al. Sequential pore wall modification in a covalent organic framework for application in lactic acid adsorption. Chem. Mater. 28, 626-631 (2016).

29. Waller, P. J. et al. Chemical conversion of linkages in covalent organic frameworks. J. Am. Chem. Soc. 138, 15519-15522 (2016).

30. Dibble, D. J. et al. An Aza-Diels-Alder route to polyquinolines. Macromolecules 48, 557-561 (2015).

31. Xu, H., Gao, J. \& Jiang, D. Stable, crystalline, porous, covalent organic frameworks as a platform for chiral organocatalysts. Nat. Chem. 7, 905 (2015). 
32. $\mathrm{Hu}, \mathrm{Y}$. et al. Probing the chemical structure of monolayer covalent-organic frameworks grown via Schiff-base condensation reactions. ChemComm 52, 9941-9944 (2016).

33. Gammon, W., Kraft, O., Reilly, A. \& Holloway, B. Experimental comparison of N (1s) X-ray photoelectron spectroscopy binding energies of hard and elastic amorphous carbon nitride films with reference organic compounds. Carbon N. Y. 41, 1917-1923 (2003).

34. Zhu, Y. et al. Unravelling surface and interfacial structures of a metal-organic framework by transmission electron microscopy. Nat. Mater. 16, 532-536 (2017).

35. Medina, D. D. et al. Oriented thin films of a benzodithiophene covalent organic framework. ACS Nano 8, 4042-4052 (2014).

36. Ascherl, L. et al. Molecular docking sites designed for the generation of highly crystalline covalent organic frameworks. Nat. Chem. 8, 310-316 (2016).

37. Peng, Y. et al. Ultrathin two-dimensional covalent organic framework nanosheets: preparation and application in highly sensitive and selective DNA detection. J. Am. Chem. Soc. 139, 8698-8704 (2017).

38. Bai, L. et al. Nanoscale covalent organic frameworks as smart carriers for drug delivery. ChemComm 52, 4128-4131 (2016).

39. Mullangi, D., Shalini, S., Nandi, S., Choksi, B. \& Vaidhyanathan, R. Superhydrophobic covalent organic frameworks for chemical resistant coatings and hydrophobic paper and textile composites. J. Mater. Chem. A 5, 8376-8384 (2017).

40. Zhang, W., Hu, Y., Ge, J., Jiang, H.-L. \& Yu, S.-H. A facile and general coating approach to moisture/water-resistant metal-organic frameworks with intact porosity. J. Am. Chem. Soc. 136, 16978-16981 (2014).

41. Nguyen, J. G. \& Cohen, S. M. Moisture-resistant and superhydrophobic metal - organic frameworks obtained via postsynthetic modification. J. Am. Chem. Soc. 132, 4560-4561 (2010).

\section{Acknowledgements}

Work at the Molecular Foundry was supported by the Office of Science, Office of Basic Energy Sciences, of the U.S. Department of Energy under Contract No. DE-AC02-

05CH11231. S.C. is grateful to the support from the National Nature Science Foundation of China (Grant No. 21603076). We thank Liana M. Klivansky and Haoran Yang for their help in structural characterizations of our samples.

\section{Author contributions}

Y.L., E.C., J.C., and J.U. conceived the project and provided funding. Y.L. designed the experiments, X.Li and X.Lei conducted the experiments, C.Z. performed TEM measurement and analysis, S.C. performed PXRD simulations and analysis, V.A. assisted with XPS measurements, F.H. assisted with PXRD measurements and Y.L., E.C., J.C., J.U., and X.Li wrote the manuscript.

\section{Additional information}

Supplementary Information accompanies this paper at https://doi.org/10.1038/s41467 018-05462-4.

Competing interests: A patent application has been filed before the submission of this manuscript. Apart from that, the authors declare no competing interests.

Reprints and permission information is available online at http://npg.nature.com/ reprintsandpermissions/

Publisher's note: Springer Nature remains neutral with regard to jurisdictional claims in published maps and institutional affiliations.

\begin{abstract}
(c) (i)
Open Access This article is licensed under a Creative Commons Attribution 4.0 International License, which permits use, sharing, adaptation, distribution and reproduction in any medium or format, as long as you give appropriate credit to the original author(s) and the source, provide a link to the Creative Commons license, and indicate if changes were made. The images or other third party material in this article are included in the article's Creative Commons license, unless indicated otherwise in a credit line to the material. If material is not included in the article's Creative Commons license and your intended use is not permitted by statutory regulation or exceeds the permitted use, you will need to obtain permission directly from the copyright holder. To view a copy of this license, visit http://creativecommons.org/ licenses/by/4.0/.
\end{abstract}

(C) The Author(s) 2018 